\title{
CXCR4 antagonist alleviates proliferation and collagen synthesis of cardiac fibroblasts induced by TGF- $\beta 1$
}

\author{
Wei Wang, Chuntao Wu, Rong Luo and Zheng Zhang \\ Department of Cardiovascular Medicine, Qingpu Branch of Zhongshan Hospital Affiliated to Fudan University, Shanghai, \\ China
}

\begin{abstract}
We aimed to investigate the effects of CX-C chemokine receptor type 4 (CXCR4) on transforming growth factor (TGF)- $\beta 1$-induced cardiac fibrosis in Human cardiac fibroblasts (HCFs). HCFs were stimulated with TGF- $\beta 1$, and the level of $\alpha$-smooth muscle actin ( $\alpha$-SMA) was assessed by immunofluorescence assay. The expression of CXCR4 was detected by Western blotting. Then the cells were incubated with CXCR4 antagonist AMD 3465. Cell viability was measured by CCK- 8 assay. The expression of $\alpha$-SMA, proliferating cell nuclear antigen (PCNA) and Ki67 were examined. Collagen synthesis was detected by sirius red staining. Moreover, the expression of phpspho-Smad2 ( $\mathrm{p}$-Smad2) and $\mathrm{p}-\mathrm{Smad} 3$ were determined. We found that the level of $\alpha$-SMA was increased after induction with TGF- $\beta 1$. The expression of CXCR 4 was upregulated in TGF- $\beta 1$-treated HCFs. Following treatment with AMD 3465, cell proliferation was inhibited coupled with a decrease in PCNA and Ki67 expression. Additionally, the expression of a-SMA was decreased after being intervened with AMD 3465. Concurrently, the levels of collagen were reduced accompanied by downregulation of Collagen I and III. Furthermore, AMD 3465 treatment decreased the expression of $\mathrm{p}-\mathrm{Smad} 2$ and $\mathrm{p}$-Smad3. Our findings suggested that CXCR4 antagonist AMD 3465 could alleviate cardiac fibrosis via blocking TGF- $\beta 1$-induced activation of Smad2/3 in HCFs.
\end{abstract}

Key words: CX-C chemokine receptor type 4 - AMD 3465 - TGF- $\beta 1$ - Cardiac fibrosis - Collagen

\section{Introduction}

Characterized by the excessive proliferation of cardiac fibroblasts and overproduction of collagen, myocardial fibrosis has been identified as a critical event in physiological and pathological cardiac remodeling, thus contributing to many cardiac dysfunctions, such as cardiac arrhythmia, myocardial infarction and heart failure (Kong et al. 2014; Rathod et al. 2016). To date, however, there is still no effective diagnosis and treatment for cardiac fibrosis. Therefore, it is of great clinical and scientific significance to explore the molecular mechanism and find out potential drug targets of myocardial fibrosis.

Correspondence to: Zheng Zhang, Cardiovascular Medicine Department, Qingpu Branch of Zhongshan Hospital Affiliated to Fudan University, 1158 Park East Road, Qingpu District, Shanghai, 201700, China

E-mail: zhengzhangshine@163.com
CX-C chemokine receptor type 4 (CXCR4) is known as a typical G-protein coupled seven-fold transmembrane receptor and one of a CXCR chemokine receptor. Accumulating evidence suggests that CXCR4 plays a significant role in a number of physiological processes (Schneider et al. 2015; Luo et al. 2016). Recent researches have confirmed that CXCR4 might be implicated in the process of fibrosis. For instance, the expression level of CXCR4 is closely related to pulmonary fibrosis in Adenosine A2a receptor (A2aR) gene-knockout mice (Chen et al. 2017). The activation of CXCR4 contributed to kidney fibrosis and genetic ablation of CXCR4 prevented unilateral ureteral obstruction induced fibrosis via inhibiting transforming growth factor (TGF)- $\beta 1$ pathways (Yuan et al. 2015). In addition, emerging evidence supports the notion that inhibition of CXCR4 could treat pulmonary fibrosis (Ding et al. 2018). Importantly, a previous study reported that activation of CXCR4 pathway leads to cardiac fibrosis in dilated cardiomyopathy (Chu et al. 2019). However, the effect of 
CXCR4 and the underlying mechanisms in human cardiac fibroblasts (HCFs) of myocardial fibrosis remains to be further elucidated.

Numerous studies unveiled that TGF- $\beta 1$ is one of the most powerful profibrogenic mediators and a dominant stimulator of collagen production (Chen et al. 2005). Therefore, in the current study, we aimed to investigate the effects of CXCR4 on TGF- $\beta 1$-induced cardiac fibrosis. As a well-known highly selective CXCR4 antagonist, AMD 3465 was used to block CXCR4 in HCFs (Hartimath et al. 2014). And our data demonstrated that CXCR4 inhibition alleviates proliferation and collagen secretion of cardiac fibroblasts in HCFs through blocking TGF- $\beta 1$-induced activation of $\operatorname{Smad} 2 / 3$.

\section{Materials and Methods}

\section{Cell culture and treatment}

HCFs were obtained from the American Type Culture Collection (ATCC; Manassas, VA, USA) and grown in DMEM (Thermo Fisher Scientific, Inc., Waltham, MA, USA) containing $10 \%$ FBS. All cells were incubated in an incubator containing $5 \% \mathrm{CO}_{2}$. HCFs were stimulated with TGF- $\beta 1$ $(20 \mathrm{ng} / \mathrm{ml})$ for $24 \mathrm{~h}$, then the cells were incubated with various concentrations of age-related macular degeneration (AMD) $3465(2.5,5$ and $10 \mu \mathrm{M})$ for $48 \mathrm{~h}$. Cells that cultured in normal conditions were used as control.

\section{Immunofluorescence assay}

The levels of $\alpha$-smooth muscle actin ( $\alpha$-SMA) and Ki67 were detected by an immunofluorescence assay. Briefly, after being fixed in $4 \%$ formaldehyde for $15 \mathrm{~min}$ and permeabilized with $0.1 \%$ Triton $^{\mathrm{Tm}} \mathrm{X}-100$ for another $15 \mathrm{~min}$, the cells were blocked in $5 \%$ goat serum for $1 \mathrm{~h}$. Subsequently, cells were probed overnight at $4^{\circ} \mathrm{C}$ with a-SMA (\#19245, cell signaling technology) or Ki67 (\#12075, cell signaling technology), followed by incubation with secondary antibodies for $1 \mathrm{~h}$. Finally, after being stained with DAPI (D9542, Sigma) for $5 \mathrm{~min}$, the samples were imaged using a fluorescence microscope (IX73-A12FL/PH; Olympus, Japan).

\section{Quantitative real-time polymerase chain reaction ( $q P C R$ )}

RNA in cells is collected using TRizol reagent (Invitrogen; Thermo Fisher Scientific, Inc.) in line with the supplier's instructions. And cDNA was obtained using SuperScript II reverse transcriptase (Thermo Fisher Scientific, Inc.). Then quantitative PCR reactions were performed using PowerUp ${ }^{\mathrm{mm}}$ SYBR Green (Thermo Fisher Scientific, Inc.) with ABI 7500 thermocycler (Thermo Fisher Scientific,
Inc. USA). Primers used were as follows: a-SMA, Forward 5'-GTGTTGCCCCTGAAGAGCAT-3' and Reverse 5'-GCTGGGACATTGAAAGTCTCA-3'; GAPDH, Forward 5'-CTGGGCTACACTGAGCACC-3' and Reverse 5'-AAGTGGTCGTTGAGGGCAATG-3'. GAPDH was used as control for normalization. The expression was calculated using the $2^{-\Delta \Delta \mathrm{Cq}}$ method.

\section{Cell counting kit-8 (CCK-8) assay}

Cell proliferation was measured by a CCK- 8 kit. HCFs were plated into 96 -well plates $\left(3 \times 10^{3}\right.$ cells/well). The cells were treated with TGF- $\beta 1$ for $24 \mathrm{~h}$ and then incubated with AMD 3465 for $48 \mathrm{~h}$. Then, $10 \mu \mathrm{l} \mathrm{CCK}-8$ (Beyotime, Beijing, China) was added into each experimental well for $2 \mathrm{~h}$. Absorbance of each well was read at a wavelength of $450 \mathrm{~nm}$ using a microplate reader (Molecular Devices, Sunnyvale, CA), and the viability of the control group was assumed to be $100 \%$.

\section{Western blotting}

Total proteins were extracted from HCFs using RIPA lysis buffer (Beyotime, Shanghai, China). The concentration of protein was detected using a BCA protein assay kit (Beyotime, Shanghai, China). Subsequently, protein (20 ug/ lane) was isolated by SDS-polyacrylamide gels (PAGE), and electrophoretically transferred onto polyvinylidene fluoride membranes. Then, all membranes were blocked by $5 \%$ skimmed milk. After being probed with primary antibodies, membranes were subsequently incubated with secondary antibodies (sc-358914, Santa Cruz). Finally, the signals of immunoblots were visualized using an enhanced chemiluminescence system (Amersham Bioscience, USA) and analyzed with ImageJ software. The protein expression was normalized to GAPDH levels.

\section{Sirius red staining}

The degree of myocardial fibrosis of HCFs was determined using Sirius red staining. Briefly, $4 \%$ paraformaldehyde was employed to fix with cells for $10 \mathrm{~min}$, followed by incubation with $0.1 \%$ Sirius Red F3B for $30 \mathrm{~min}$. Then the cells were stained with hematoxylin and dehydrating thrice in $100 \%$ ethanol.

\section{Statistical analysis}

All quantitative data were expressed as the mean \pm standard error of the mean (mean \pm SD). Statistical analyses were carried out by using Student's unpaired $t$-test and one-way ANOVA followed by Dunnett's post-hoc test. A value of $p<$ 0.05 was considered statistically significant and each experiment was repeated at least three times. 


\section{Results}

AMD 3465 treatment inhibited the expression of CXCR4 induced by TGF- $\beta 1$ in HCFs

TGF- $\beta 1$ was employed to treat HCFs, and the level of a-SMA was determined using immunofluorescence assay in our study. As presented in Figure 1, there was almost no expression of $\alpha$-SMA in the control group. On the contrary, TGF- $\beta 1$ treatment increased the level of $\alpha$-SMA, which is a marker of myofibroblast. As a novel antagonist of CXCR4, AMD 3465 was applied to treat cells with different doses $(2.5,5$ and $10 \mu \mathrm{M})$. Proliferation of cells was measured by a CCK-8 kit. As exhibited in Figure 2A, AMD 3465 significantly reduced the proliferation of HCFs in a dosedependent manner. Moreover, when the concentration of AMD 3465 was $5 \mu \mathrm{M}$, the cell activity of HCFs was close to $50 \%$ compared with the control group. Take it into account, $5 \mu \mathrm{M}$ AMD 3465 treatment was chosen for the following experiment. It was found that the expression of CXCR4 was enhanced in TGF- $\beta 1$-induced HCFs and AMD 3465 decreased the expression of CXCR4 induced by TGF- $\beta 1$ (Fig. 2B). These results indicated that AMD 3465 treatment inhibited the expression of CXCR 4 induced by TGF- $\beta 1$ in HCFs.
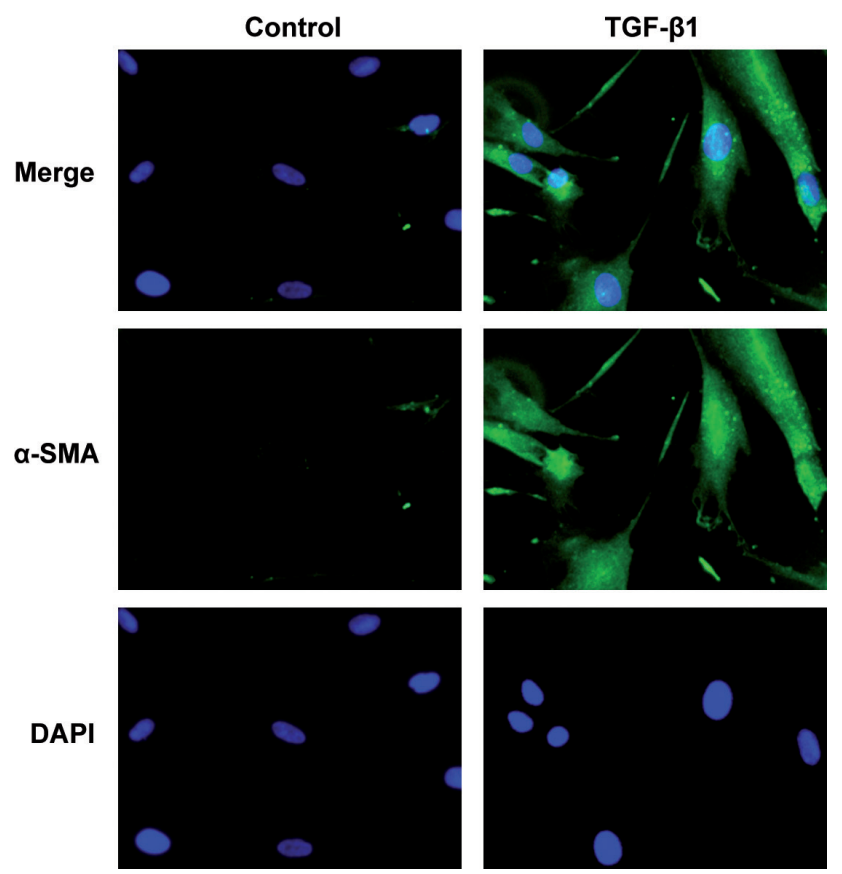

Figure 1. The expression of $\alpha$-SMA in TGF- $\beta 1$-induced human cardiac fibroblasts. Immunofluorescence staining assay was applied to measure the level of $\alpha$-SMA in each group. $200 \times$ magnification. $\alpha$-SMA, $\alpha$-smooth muscle actin; TGF- $\beta 1$, transforming growth factor $-\beta 1$.
AMD 3465 treatment relieved cell proliferation of HCFs induced by TGF- $\beta 1$

To explore the effect of AMD 3465 on cell proliferation of HCFs induced by TGF- $\beta 1$. The expression of PCNA and Ki67 were assessed in the current study. We found that TGF- $\beta 1$ upregulated the level of PCNA, whereas AMD 3465 treatment decreased this increase obviously (Fig. 3A). Concurrently, the level of Ki67 was detected by immunofluorescence assay.

A

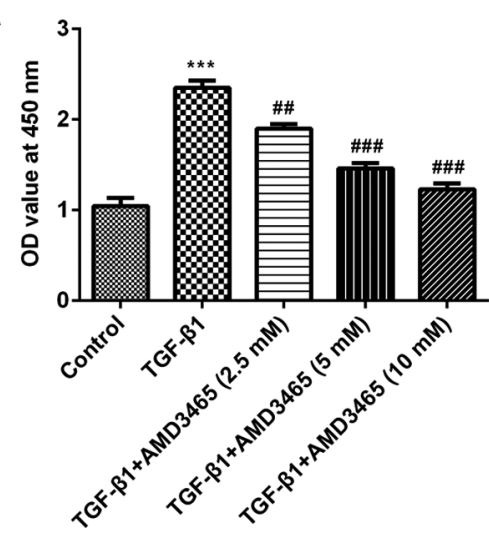

B
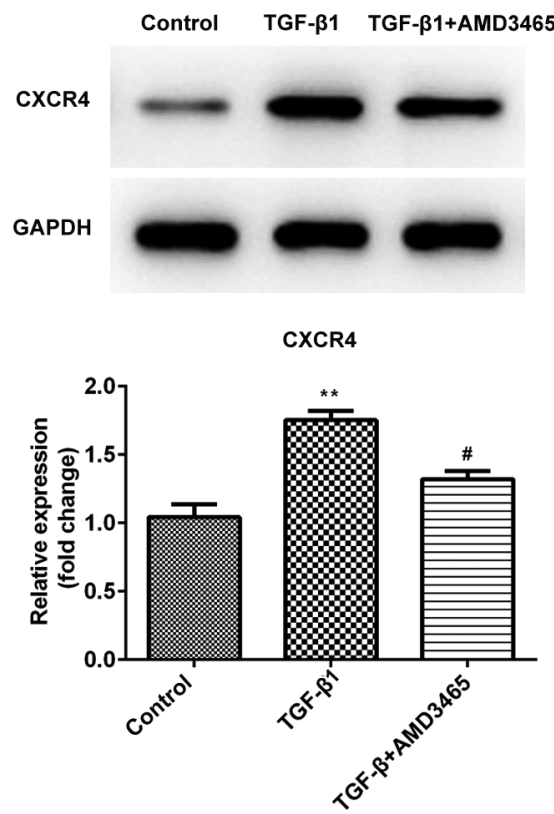

Figure 2. AMD 3465 treatment reduced cell proliferation and decreased the level of CXCR4 in TGF- $\beta 1$-treated human cardiac fibroblasts (HCFs). A. Cell proliferation was assessed by CCK-8 assay after treatment with AMD 3465 in TGF- $\beta 1$-induced HCFs. B. AMD 3465 treatment reduced the level of CXCR4 in TGF$\beta 1$-treated HCFs. ${ }^{* *} p<0.01,{ }^{* * *} p<0.001$ vs. Control; ${ }^{\#} p<0.05$, ${ }_{\# \#} p<0.01,{ }^{\# \# \#} p<0.001$ vs. TGF- $\beta 1$. TGF- $\beta 1$, transforming growth factor- $\beta 1$; CXCR4, CX-C chemokine receptor type 4 . 
As presented in Figure 3B, AMD 3465 treatment reversed the upregulation of Ki67 induced by TGF- $\beta 1$ stimulation in HCFs. These findings suggested that AMD 3465 treatment relieved cell proliferation of HCFs induced by TGF- $\beta 1$.

\section{AMD 3465 treatment alleviated TGF- $\beta 1$-induced} differentiation of HCFs

To explore the effect of AMD 3465 on TGF- $\beta 1$-induced differentiation of HCFs, the level of myofibroblast marker
a-SMA was assessed using Western blotting and immunofluorescence assay, respectively. As exhibited in Figure 4A, the level of a-SMA was increased notably in comparison with the control group, whereas AMD 3465 treatment markedly reduced TGF- $\beta 1$-induced $\alpha$-SMA expression. And the results of analysis by immunofluorescence assay was in accordance with the western blotting (Fig. 4B). These observations revealed that AMD 3465 treatment reversed the upregulated expression of $\alpha$-SMA induced by TGF- $\beta 1$ stimulation in HCFs.
A

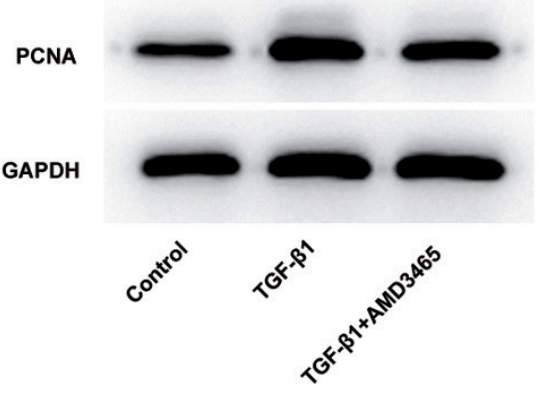

B
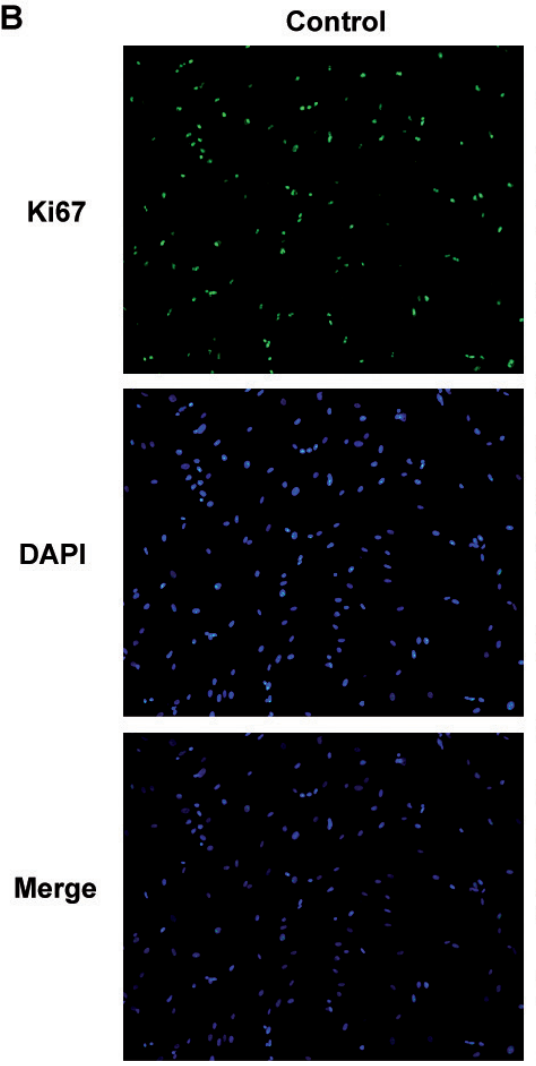

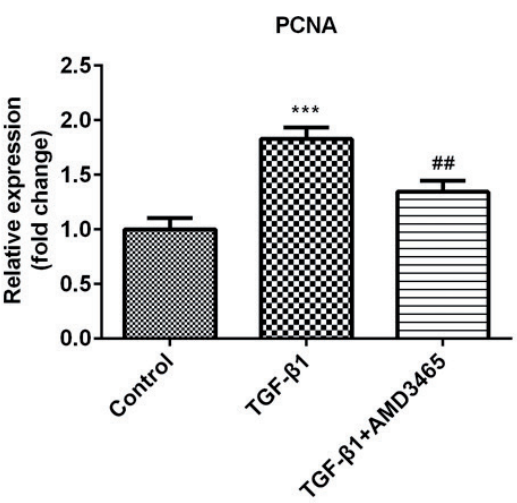

TGF- $\beta 1$
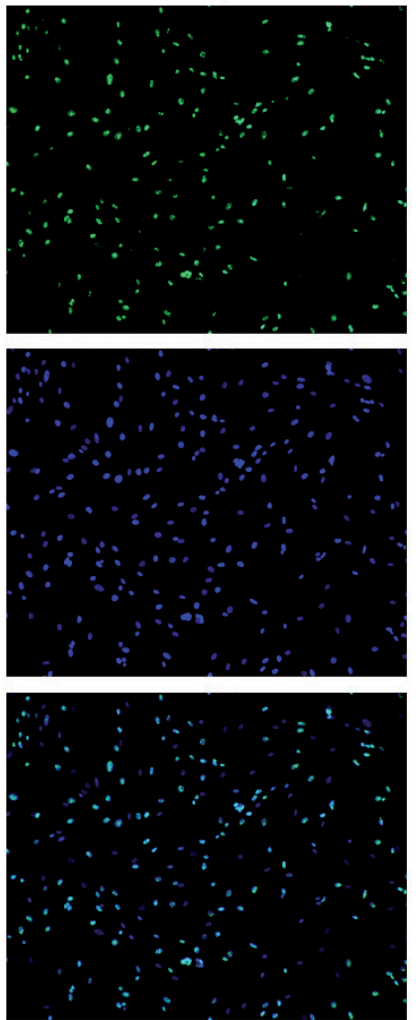

TGF- $\beta 1+A M D 3465$
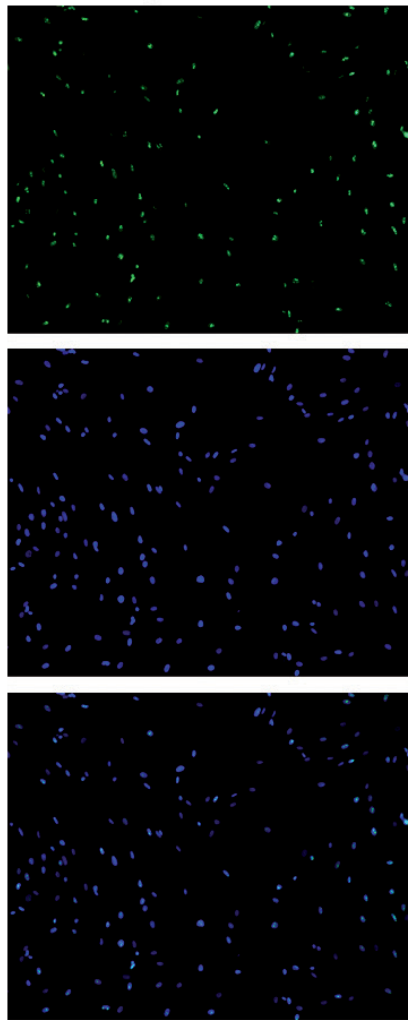

Figure 3. AMD 3465 treatment downregulated the expression of PCNA and Ki67 in TGF- $\beta 1$-induced human cardiac fibroblasts. A. The expression of PCNA was assessed by western blotting. B. The level of Ki67 was determined by immunofluorescence assay. ${ }^{* * *} p<0.001 v s$. Control; ${ }^{\# \#} p<0.01$ vs. TGF- $\beta 1.100 \times$ magnification. TGF- $\beta 1$, transforming growth factor- $\beta 1$; PCNA, proliferating cell nuclear antigen. 
AMD 3465 treatment inhibited TGF- $\beta 1$-induced collagen synthesis in HCFs

Collagen synthesis is crucial in the process of myocardial fibrosis, therefore, the expression of Collagen I and III was detected using Western blotting. As expected, the expression of Collagen I and Collagen III were increased obviously in TGF- $\beta 1$-induced HCFs. And AMD 3465 treatment dramatically suppressed both Collagen I and III expression induced by TGF- $\beta 1$ (Fig. 5A). Consistent with that, the results of sirius red staining showed that AMD 3465 markedly inhibited collagen synthesis of HCFs (Fig. 5B and C).

\section{AMD 3465 treatment blocked TGF- $\beta 1$-induced activation of Smad2/3 in HCFs}

To further investigate the potential molecular mechanism of AMD 3465 functions in myocardial fibrosis, western blotting assay was applied to detect the expression level of
A

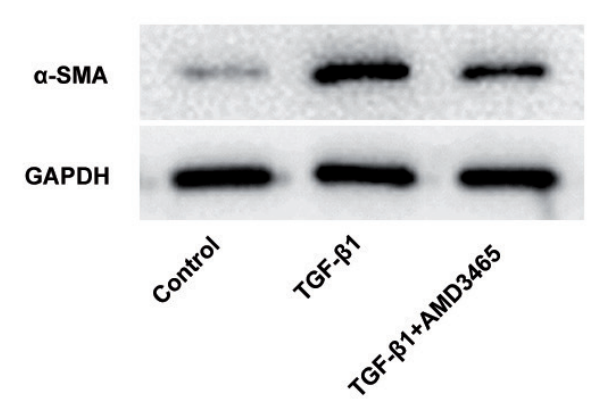

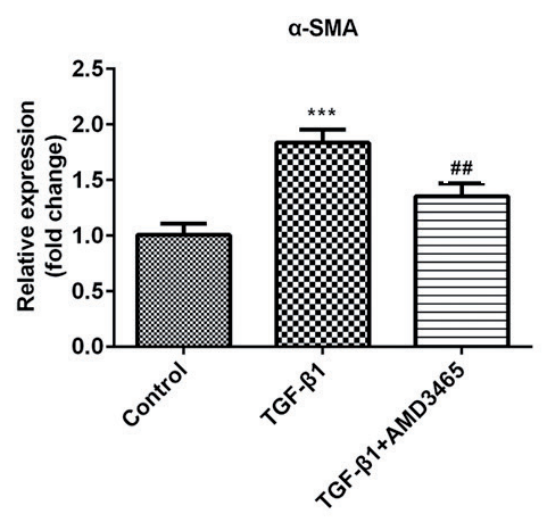

B
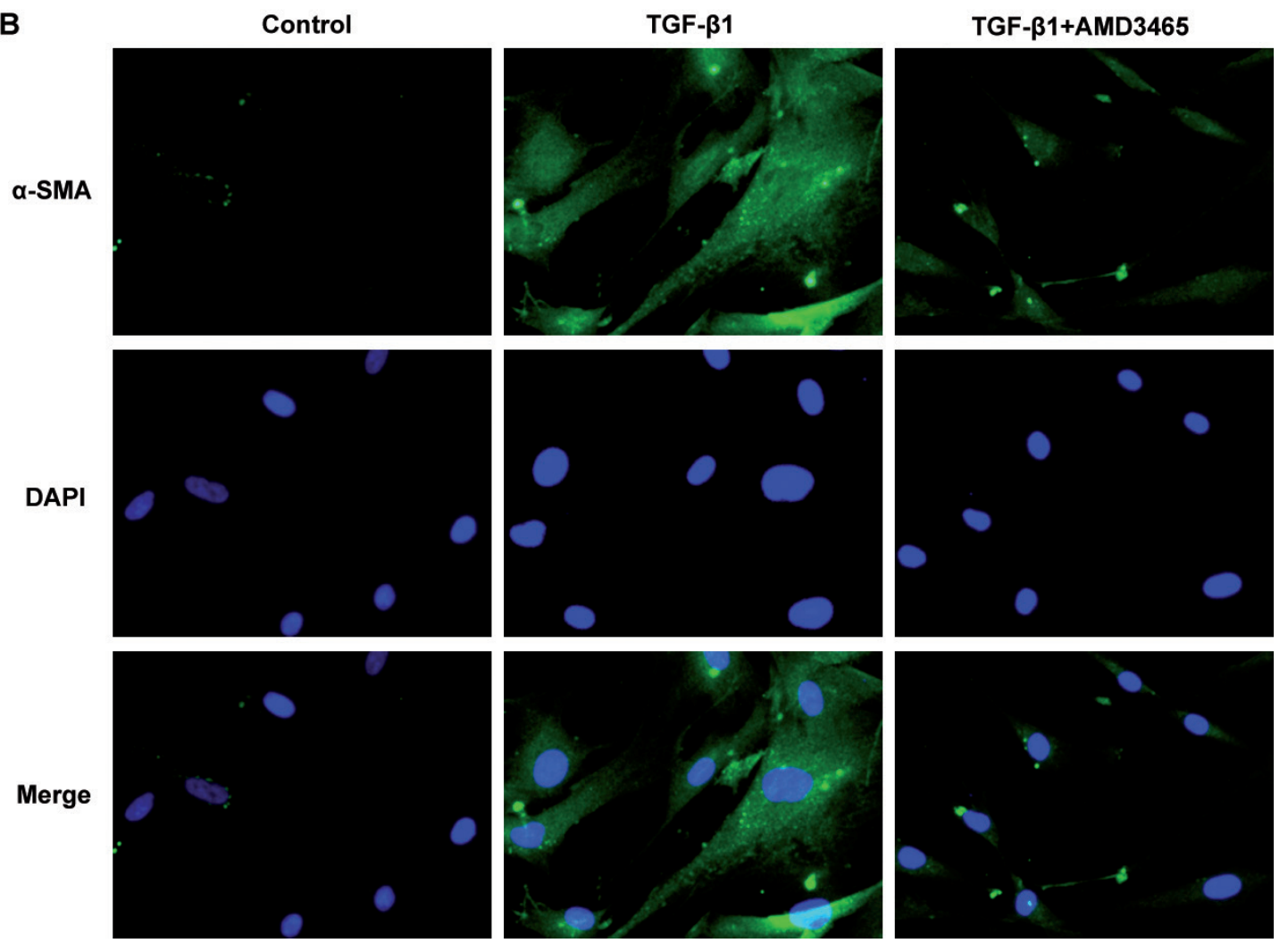

Figure 4. AMD 3465 treatment decreased the level of $\alpha$-SMA in TGF- $\beta 1$ treated human cardiac fibroblasts. The expression of $\alpha$-SMA was detected by (A) Western blotting and (B) immunofluorescence assay. ${ }^{* * *} p<0.001 v$ s. Control; ${ }^{\# \#} p<0.01 v$ s. TGF- $\beta 1.200 \times$ magnification. $\alpha$-SMA, $\alpha$-smooth muscle actin; TGF- $\beta 1$, transforming growth factor- $\beta 1$. 
A

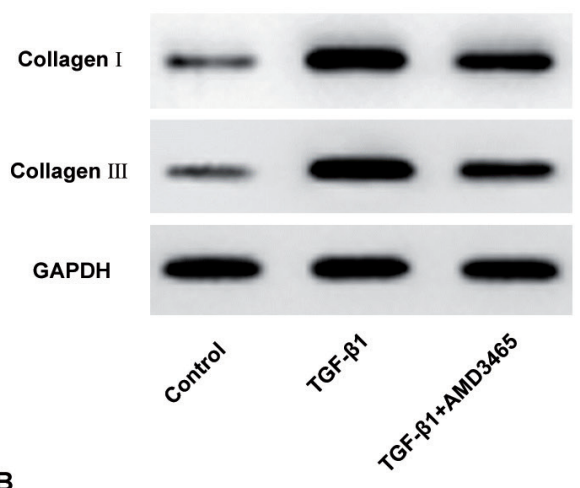

B

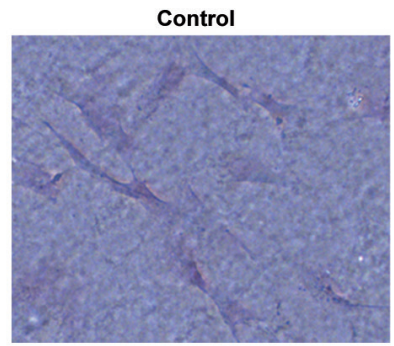

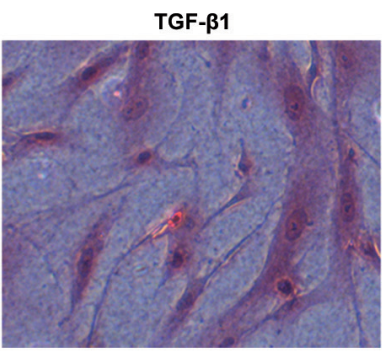

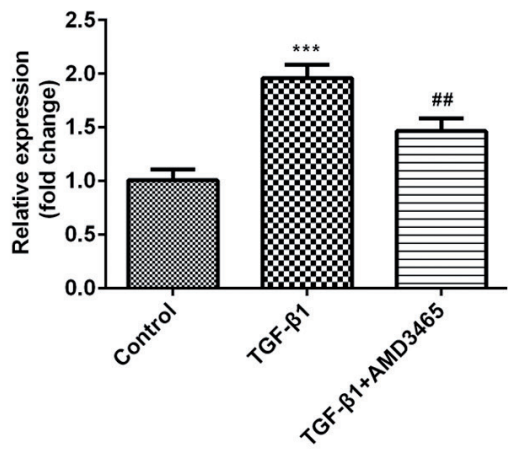

TGF-ß1+AMD3465

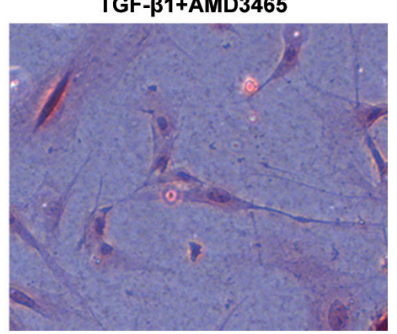

Collagen III

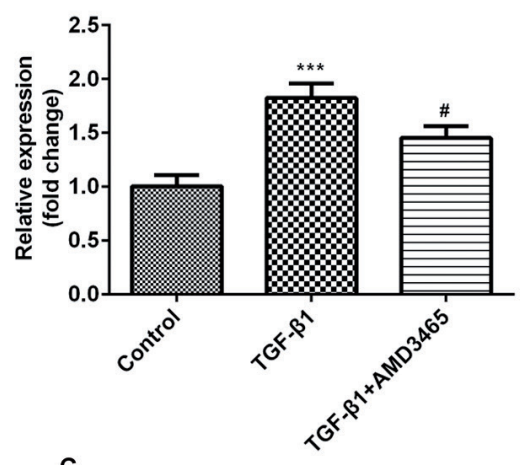

C

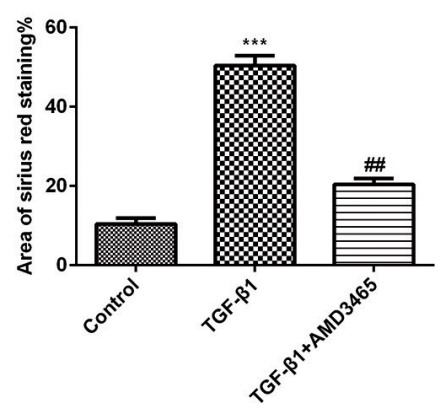

Figure 5. AMD 3465 treatment decreased the collagen secretion in TGF- $\beta 1$-treated human cardiac fibroblasts. A. The expression of Collagen I and Collagen III were evaluated by Western blotting. B. Representative images of Sirius red staining from each experimental group. C. Quantification of Sirius red staining. ${ }^{* *} p<0.001 v s$. Control; ${ }^{\#} p<0.05,{ }^{\# \#} p<0.01 v s$. TGF- $\beta 1$. TGF- $\beta 1$, transforming growth factor- $\beta 1$.

the key proteins of TGF- $\beta 1 /$ Smad signaling pathway. Our results suggested that the expression of both phpspho-Smad2 (p-Smad2) and p-Smad3 were markedly upregulated in TGF- $\beta 1$-treated HCFs (Fig. 6). And AMD 3465 treatment markedly downregulated the levels of $\mathrm{p}-\mathrm{Smad} 2$ and $\mathrm{p}-\mathrm{Smad} 3$ induced by TGF- $\beta 1$ in HCFs. Overall, above data suggested that AMD 3465 treatment could block TGF- $\beta 1$-induced activation of Smad2/3 in HCFs.
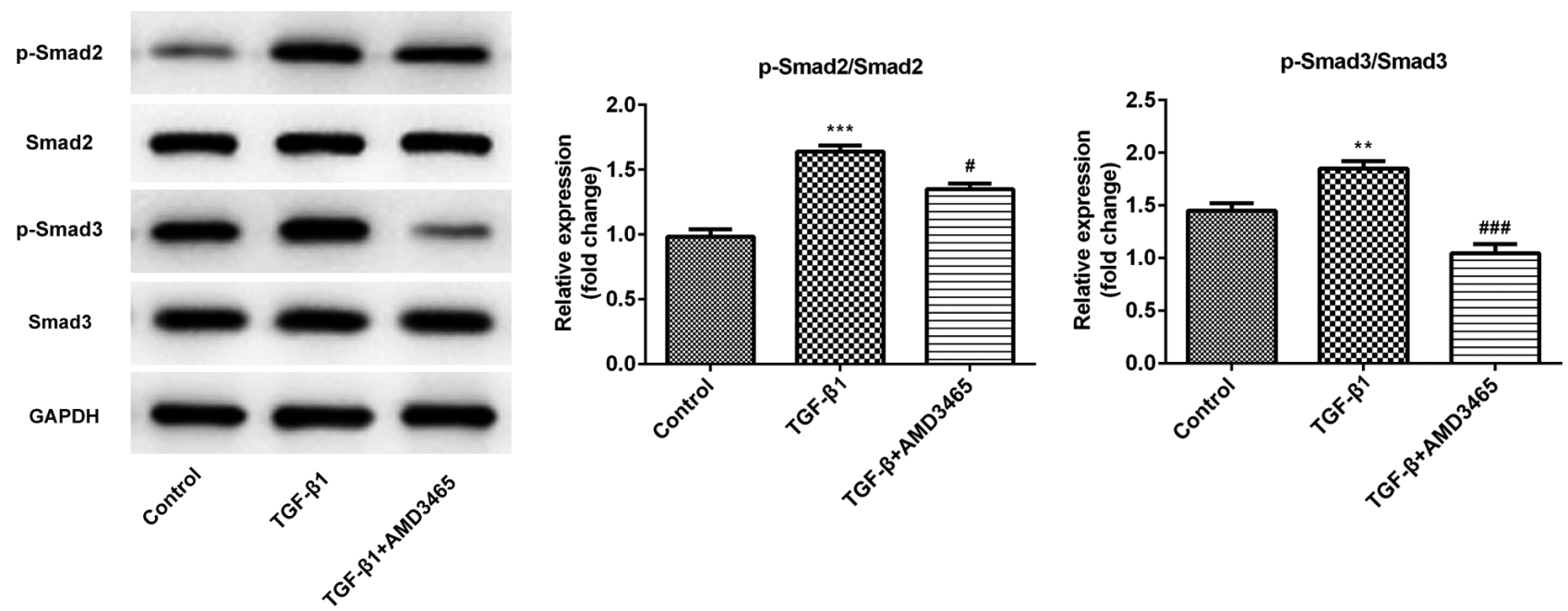

Figure 6. AMD 3465 treatment inhibited the expression of $\mathrm{p}$-Smad 2 and $\mathrm{p}$-Smad3. The levels of $\mathrm{p}$-Smad 2 and $\mathrm{p}$-Smad 3 were assessed by Western blotting. ${ }^{* *} p<0.01,{ }^{* *} p<0.001 v$ s. Control; ${ }^{\#} p<0.05,{ }^{\# \#} p<0.001 v s$. TGF- $\beta 1$. TGF- $\beta 1$, transforming growth factor- $\beta 1$; p-Smad2, phospho-Smad2. 


\section{Discussion}

CXCR4 is involved in many physiological and pathological processes including occurrence and development of cardiovascular disease (Segret et al. 2007; Chatterjee et al. 2017). Cardiac fibrosis is one of the important pathophysiological mechanisms underlying cardiovascular disease. However, little is known about the role of CXCR4 in the process of myocardial fibrosis. Recently, a study in myocardial infarction showed that CXCR4 blockade could reduce fibrosis and enhance cardiac function and survival after myocardial infarction, suggesting that CXCR4 may participate in myocardial fibrosis (Jujo et al. 2010). Herein, we aimed to investigate the effects of CXCR4 on cardiac fibroblasts. Our results showed that the expression of CXCR4 was upregulated notably in TGF- $\beta 1$-stimulated HCFs, which suggested that CXCR4 may be involved in the TGF- $\beta 1$-induced cardiac fibrosis. To further investigate the potential role of CXCR4 and reveal the underlying molecular mechanisms, the CXCR4 antagonist AMD 3465 was used to perform. We found that AMD 3465 alleviates TGF- $\beta 1$-induced collagen synthesis and myofibroblasts differentiation of HCFs via suppression of TGF- $\beta 1 / \mathrm{Smad}$ pathway. These findings suggested that CXCR4 is crucial for cardiac fibrosis and it could be a potential target in cardiac fibrosis treatment.

It has been documented previously that CXCR4 could promote the differentiation of fibroblasts to myofibroblasts (Abu El-Asrar et al. 2008; Gharaee-Kermani et al. 2012; Marts et al. 2019). For example, a study involving idiopathic pulmonary fibrosis (IPF) revealed that the expression of CXCR4 mRNAs were decreased in IPF patients compared with the healthy control (Antoniou et al. 2010). In addition, prior report has demonstrated that CXCR4 inhibition could treat the activation of HSC-T6 cells induced by TGF- $\beta 1$ in liver fibrosis (Ullah et al. 2019). Moreover, it has been well reported that CXCL4 promotes the process of cell fibrosis by inducing the $\alpha$-SMA production (Gillen et al. 2013). In our study, it was found that AMD 3465 treatment decreased the expression of $\alpha$-SMA, which was in accordance with the previous study (Xu et al. 2016).

Excessive proliferation of cardiac fibroblasts contributes to the pathological changes of fibrotic diseases. It has been reported that endogenous $\mathrm{SO}_{2}$ could inhibit myocardial fibroblast proliferation via inhibiting ERK signaling pathway (Zhang et al. 2018). Relaxin is able to reduce the expression of PCNA and reverses cardiac and renal fibrosis in rats (Lekgabe et al. 2005). In addition, a previous study reported that Dasatinib could inhibit Ki67 level and relieve cardiac fibrosis in mice induced by pressure overload (Balasubramanian et al. 2015). Consistent with these data, the present study revealed that AMD 3465 treatment decreased the proliferation of HCFs induced by TGF- $\beta 1$ accompanied by downregulation of PCNA and Ki67 expression, which are the proliferation associated genes. Our results suggested that AMD 3465 treatment relieved cell proliferation of HCFs induced by TGF- $\beta 1$. Moreover, TGF- $\beta 1$ is a multifunctional cytokine and one of the most powerful stimuli of fibroblast activation and fibrosis identified to date (Fix et al. 2019). A growing body of evidence confirms that suppression of Collagen I and III attenuates cardiac fibroblasts (Wang et al. 2018; Song and Ren 2019). We found that AMD 3465 treatment decreased the level of collagen deposition, coupled with downregulation of Collagen I and III expression. These observations revealed that AMD 3465 treatment inhibited TGF- $\beta 1$-induced collagen deposition in HCFs.

The activation of CXCR4 can induce a range of physiological responses and gene expression by activating downstream pathways (Ding et al. 2019). To investigate the precise mechanism of CXCR4 blocking in fibrosis inhibition, we assessed the level of key proteins in TGF- $\beta 1 / \mathrm{Smad}$ signaling pathway. Our data showed that AMD 3465 markedly downregulated the levels of p-Smad2 and p-Smad3 induced by TGF- $\beta 1$ in HCFs. Those results suggested that there was a crosstalk between CXCR 4 and TGF- $\beta 1 /$ Smad pathway. Based on the above results, we concluded that AMD 3465 treatment could block TGF- $\beta 1$-induced activation of Smad2/3 in HCFs.

In conclusion, our data demonstrated that CXCR4 antagonist AMD 3465 alleviates cardiac fibrosis induced by TGF- $\beta 1$ in HCFs via blocking, at least partly, Smad dependent signaling pathway. TGF- $\beta 1$ obviously increases the level of CXCR4, and AMD 3465 blocks TGF- $\beta 1$-induced activation of Smad2/3 in HCFs. Interference of CXCR 4 may be a potential treatment for myocardial fibrosis.

\section{References}

Abu El-Asrar AM, Struyf S, Van Damme J, Geboes K (2008): Circulating fibrocytes contribute to the myofibroblast population in proliferative vitreoretinopathy epiretinal membranes. Br. J. Ophthalmol. 92, 699-704

https://doi.org/10.1136/bjo.2007.134346

Antoniou KM, Soufla G, Lymbouridou R, Economidou F, Lasithiotaki I, Manousakis M, Drositis I, Spandidos DA, Siafakas NM (2010): Expression analysis of angiogenic growth factors and biological axis CXCL12/CXCR4 axis in idiopathic pulmonary fibrosis. Connect Tissue Res. 51, 71-80 https://doi.org/10.3109/03008200903056150

Balasubramanian S, Pleasant, Kasiganesan H, Quinones L, Zhang Y, Sundararaj KP, Roche S, O`Connor R, Bradshaw AD, Kuppuswamy D (2015): Dasatinib attenuates pressure overload induced cardiac fibrosis in a murine transverse aortic constriction model. PLoS One 10, e0140273 https://doi.org/10.1371/journal.pone.0140273

Chatterjee M, Rath D, Schlotterbeck J, Rheinlaender J, WalkerAllgaier B, Alnaggar N, Zdanyte M, Muller I, Borst O, Geisler T, 
et al. (2017): Regulation of oxidized platelet lipidome: implications for coronary artery disease. Eur. Heart J. 38, 1993-2005 https://doi.org/10.1093/eurheartj/ehx146

Chen MH, Chen JC, Tsai CC, Wang WC, Chang DC, Tu DG, Hsieh HY (2005): The role of TGF-beta 1 and cytokines in the modulation of liver fibrosis by Sho-saiko-to in rat's bile duct ligated model. J. Ethnopharmacol. 97, 7-13 https://doi.org/10.1016/j.jep.2004.09.040

Chen Y, Yu X, He Y, Zhang L, Huang X, Xu X, Chen M, Chen X, Wang L (2017): Activation of A2aR attenuates bleomycininduced pulmonary fibrosis via the SDF-1/CXCR4 axis-related pathway. Am. J. Transl. Res. 9, 4125-4136

Chu PY, Joshi MS, Horlock D, Kiriazis H, Kaye DM (2019): CXCR4 antagonism reduces cardiac fibrosis and improves cardiac performance in dilated cardiomyopathy. Front. Pharmacol. 10, 117 https://doi.org/10.3389/fphar.2019.00117

Ding L, Zhu C, Yu F, Wu P, Chen G, Ullah A, Wang K, Sun M, Li J, Oupicky D (2018): Pulmonary delivery of polyplexes for combined PAI-1 gene silencing and CXCR4 inhibition to treat lung fibrosis. Nanomedicine 14, 1765-1776 https://doi.org/10.1016/j.nano.2018.05.005

Ding Q, Sun J, Xie W, Zhang M, Zhang C, Xu X (2019): Stemona alkaloids suppress the positive feedback loop between M2 polarization and fibroblast differentiation by inhibiting JAK2/ STAT3 pathway in fibroblasts and CXCR4/PI3K/AKT1 pathway in macrophages. Int. Immunopharmacol. 72, 385-394 https://doi.org/10.1016/j.intimp.2019.04.030

Fix C, Carver-Molina A, Chakrabarti M, Azhar M, Carver W (2019): Effects of the isothiocyanate sulforaphane on TGFbeta1-induced rat cardiac fibroblast activation and extracellular matrix interactions. J. Cell Physiol. 234, 13931-13941 https://doi.org/10.1002/jcp.28075

Gharaee-Kermani M, Kasina S, Moore BB, Thomas D, Mehra R, Macoska JA (2012): CXC-type chemokines promote myofibroblast phenoconversion and prostatic fibrosis. PLoS One 7, e49278 https://doi.org/10.1371/journal.pone.0049278

Gillen JR, Zhao Y, Harris DA, Lapar DJ, Stone ML, Fernandez LG, Kron IL, Lau CL (2013): Rapamycin blocks fibrocyte migration and attenuates bronchiolitis obliterans in a murine model. Ann. Thorac. Surg. 95, 1768-1775

https://doi.org/10.1016/j.athoracsur.2013.02.021

Hartimath SV, van Waarde A, Dierckx RA, de Vries EF (2014): Evaluation of N-[(11)C]methyl-AMD3465 as a PET tracer for imaging of CXCR4 receptor expression in a C6 glioma tumor model. Mol. Pharm. 11, 3810-3817 https://doi.org/10.1021/mp500398r

Jujo K, Hamada H, Iwakura A, Thorne T, Sekiguchi H, Clarke T, Ito A, Misener S, Tanaka T, Klyachko E, et al. (2010): CXCR4 blockade augments bone marrow progenitor cell recruitment to the neovasculature and reduces mortality after myocardial infarction. Proc. Natl. Acad. Sci. USA 107, 11008-11013 https://doi.org/10.1073/pnas.0914248107

Kong P, Christia P, Frangogiannis NG (2014): The pathogenesis of cardiac fibrosis. Cell. Mol. Life Sci. 71, 549-574 https://doi.org/10.1007/s00018-013-1349-6

Lekgabe ED, Kiriazis H, Zhao C, Xu Q, Moore XL, Su Y, Bathgate RA, Du XJ, Samuel CS (2005): Relaxin reverses cardiac and renal fibrosis in spontaneously hypertensive rats. Hypertension $46,412-418$ https://doi.org/10.1161/01.HYP.0000171930.00697.2f

Luo X, Tai WL, Sun L, Pan Z, Xia Z, Chung SK, Cheung CW (2016): Crosstalk between astrocytic CXCL12 and microglial CXCR4 contributes to the development of neuropathic pain. Molecular pain 12, 1-15 https://doi.org/10.1177/1744806916636385

Marts LT, Guidot DM, Sueblinvong V (2019): HIV-1 protein gp120 induces mouse lung Fibroblast-to-myofibroblast transdifferentiation via CXCR4 activation. Am. J. Med. Sci. 357, 483-491 https://doi.org/10.1016/j.amjms.2019.03.006

Rathod RH, Powell AJ, Geva T (2016): Myocardial fibrosis in congenital heart disease. Circ. J. 80, 1300-1307 https://doi.org/10.1253/circj.CJ-16-0353

Schneider T, Ehrig K, Liewert I, Alban S (2015): Interference with the CXCL12/CXCR4 axis as potential antitumor strategy: superiority of a sulfated galactofucan from the brown alga Saccharina latissima and fucoidan over heparins. Glycobiology 25, 812-824 https://doi.org/10.1093/glycob/cwv022

Segret A, Rucker-Martin C, Pavoine C, Flavigny J, Deroubaix E, Chatel MA, Lombet A, Renaud JF (2007): Structural localization and expression of CXCL12 and CXCR4 in rat heart and isolated cardiac myocytes. J. Histochem. Cytochem. 55, 141-150 https://doi.org/10.1369/jhc.6A7050.2006

Song H, Ren J (2019): Protocatechuic acid attenuates angiotensin II-induced cardiac fibrosis in cardiac fibroblasts through inhibiting the NOX4/ROS/p38 signaling pathway. Phytother. Res. 33, 2440-2447 https://doi.org/10.1002/ptr.6435

Ullah A, Wang K, Wu P, Oupicky D, Sun M (2019): CXCR4-targeted liposomal mediated co-delivery of pirfenidone and AMD3100 for the treatment of TGFbeta-induced HSC-T6 cells activation. Int. J. Nanomedicine 14, 2927-2944 https://doi.org/10.2147/IJN.S171280

Wang Z, Stuckey DJ, Murdoch CE, Camelliti P, Lip GYH, Griffin M (2018): Cardiac fibrosis can be attenuated by blocking the activity of transglutaminase 2 using a selective small-molecule inhibitor. Cell Death Dis. 9, 613

https://doi.org/10.1038/s41419-018-0573-2

Xu Y, Zhang Q, Xue W, Zeng S, Zhang Z, Zhang X, Hu X (2016): CXC Chemokine Receptor 4 (CXCR4) antagonist, a novel pathway to prevent chronic allograft nephropathy. Ann. Transplant. 21, 728-734 https://doi.org/10.12659/AOT.899492

Yuan A, Lee Y, Choi U, Moeckel G, Karihaloo A (2015): Chemokine receptor Cxcr4 contributes to kidney fibrosis via multiple effectors. Am. J. Physiol. Renal Physiol. 308, F459-472 https://doi.org/10.1152/ajprenal.00146.2014

Zhang LL, Du JB, Tang CS, Jin HF, Huang YQ (2018): Inhibitory effects of sulfur dioxide on rat myocardial fibroblast proliferation and migration. Chin. Med. J. (Engl) 131, 1715-1723 https://doi.org/10.4103/0366-6999.235875

Received: September 5, 2019

Final version accepted: November 4, 2019 